\title{
Temporal interference effects in auditory amplitude discrimination'
}

ROBERT D. SORKIN ${ }^{2}$

SENSORY INTELLIGENCE LABORATORY, THE UNIVERSITY OF MICHIGAN

The efficiency, $\eta$, of performance in amplitude discrimina tion is examined as a function of the temporal separation, $\tau$, of the two signals to be discriminated. Performance in a monaural amplitude discrimination task is compared with that in a dichotic amplitude discrimination task, in which the first of the two signals was always presented to one ear and the second signal to the other ear. The difference in the shape of the resulting $\eta$ versus 5 functions for the monaural and dichotic cases is interpreted in terms of peripheral and central interference effects

Various kinds of auditory experiments have demonstrated that performance decrements occur when an observer must process an input arriving in close time proximity to another input. Indeed, such interference effects appear to be quite general in visual signal processing and short-term memory situations. Monaural studies of amplitude discrimination and monaural and dichotic experiments on forward and backward masking phenomena are two illustrations of the effect. It may be possible to interpret such phenomena with the notion that the auditory system behaves much like a tuned narrow band filter; indeed, much of the narrow band character of the system has been attributed to the transducer operation of the end organ. The present study is an attempt to evaluate some of these interpretations with the additional goal of perhaps isolating the peripheral filter-like characteristics from more "central" ones.

In a study of the memory requirements of the human observer in auditory detection and discrimination, Tanner (1961) obtained functions showing how the efficiency of amplitude discrimination performance is a function of the time, $r$, between the ending times of the signal pulses to be discriminated. For $\tau$ greater than about $500 \mathrm{msec}_{\circ}$, this short term amplitude memory function drops off as a function of time. Figure 1 is based on his data. Equally interesting, however, is the expected result that as the time separation between the pulses reaches zero, performance sharply drops off to chance. The reason that the result was expected is that some sort of transducer operation must be performed on the inputs to the system, and the transducer operation may be comparable to that of a narrow band filter. The output of such a filter, given a signal input, may be extended longer than the original duration of the input. Hence the input is spread over a longer time period, and signals arriving close in time tend to be confused. Figure 1 shows Tanner's attempt to fit this effect with a simple transducer model. It seems evident that all of the selective or narrow band character of the auditory system should not necessarily exist at the end organ transducer. Rather, some degree of narrow band processing should also occur later on in the system, perhaps at a more "central" level. A type of task was sought which

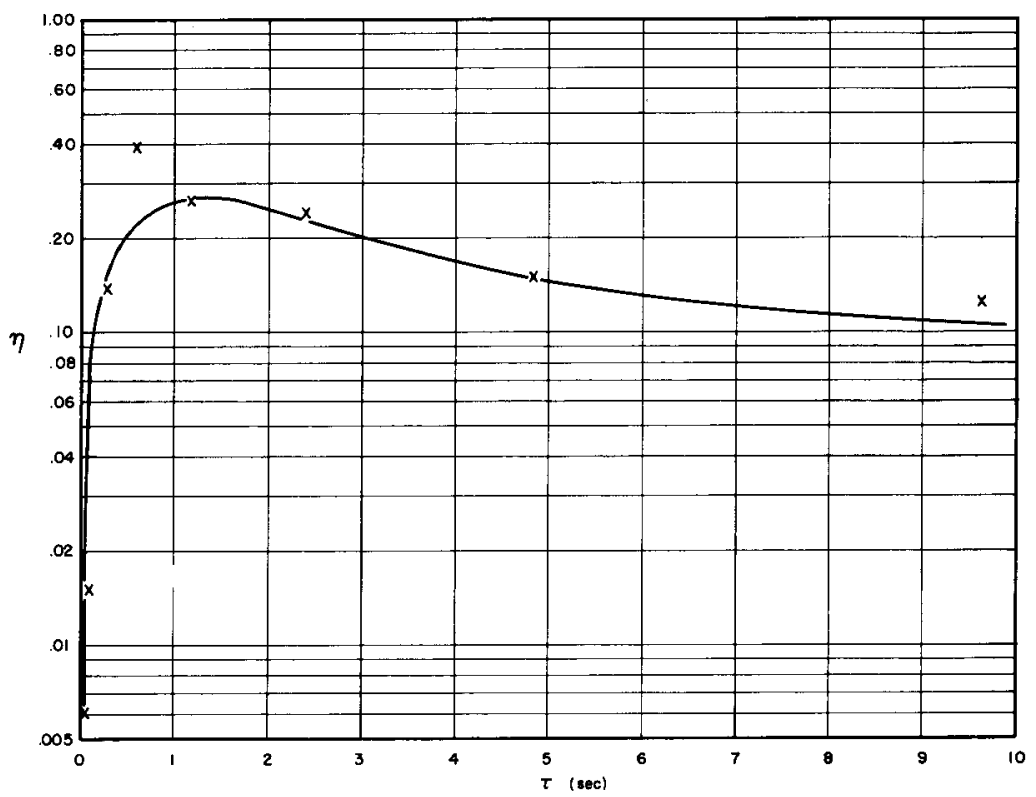

Fig. 1. The observer efficiency, $\eta$, in monaural amplitude discrimination as a function of the time interval, $\tau$, between the ending time of the first signal and the ending time of the second. 


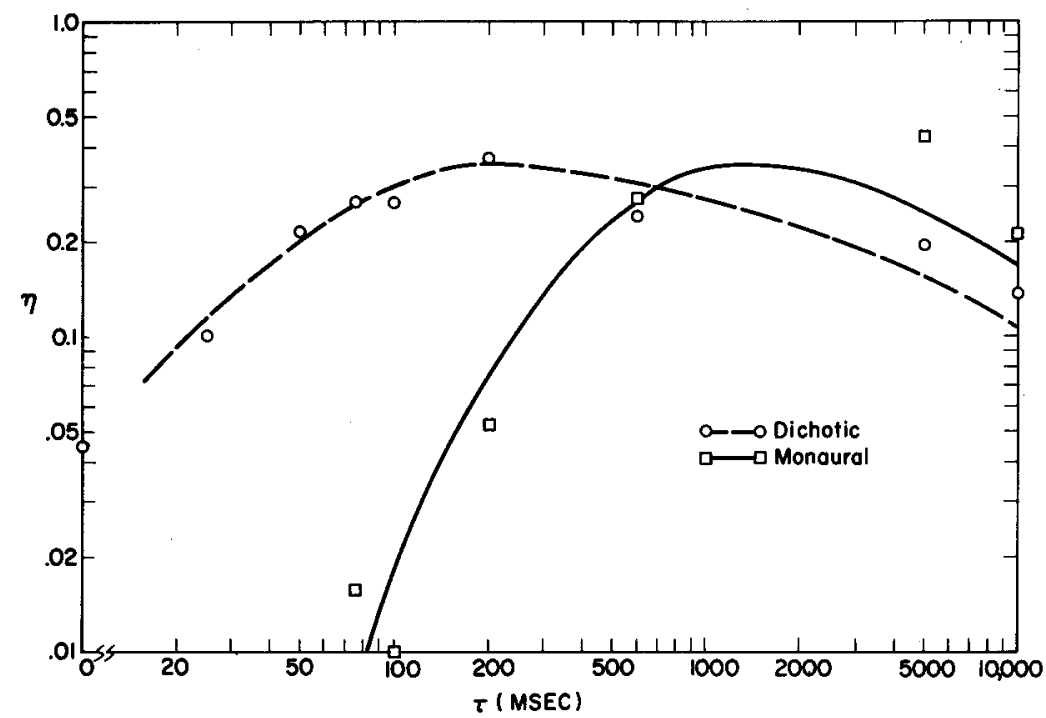

Fig. 2. The efficiency, $\eta$, of observer 1 as a function of the time interval, $\tau$, jetween the ending time of the first signal and the ending time of the second.

might possibly isolate the end organ filter effect from the more central filter effects. The term filter, in this context, is employed to describe the very general types of selective processes which may be involved in the auditory system. A likelihood-ratio computer is an example of a mathematically definable filter, since such a computation involves the selection of information which is relevant to particular decisions. A linear passive filter of the sort found in tuned audio frequency circuits is a type of filter which is often useful in describing the peripheral auditory system. Thus when one studies the filter characteristics of the peripheral auditory system, one expects to find time constants which reflect the energy storage and decay characteristics of the latter type of filter. It may be quite reasonable, however, to expect to find similar time constant effects in filters defined as broadly as at the outset. Hence temporal interference effects might be observed as characteristics of the more central selective processes.
A procedure for eliminating the end organ filter effects would involve presenting two short signals close together in time, but somehow isolating the signals from each other until it is necessary for the central processing mechanism to become involved. The procedure arrived at is simply a dichotic version of the task of amplitude discrimination using the twoalternative temporal forced-choice medhod. The two observation intervals are isolated by having each interval occur always in a specified ear. Thus, for example, the first observation interval always occurs in the left ear and the second in the right. The task is then to report which interval, that is, which ear, contains the larger amplitude signal. The response of the auditory system may now be evaluated at extremely small time separations between the inputs, since complete overlap of the inputs is now equivalent to a lateralization task, where above chance performance will still be expected. The situation can be reproduced in the monaural case, in order to allow comparison

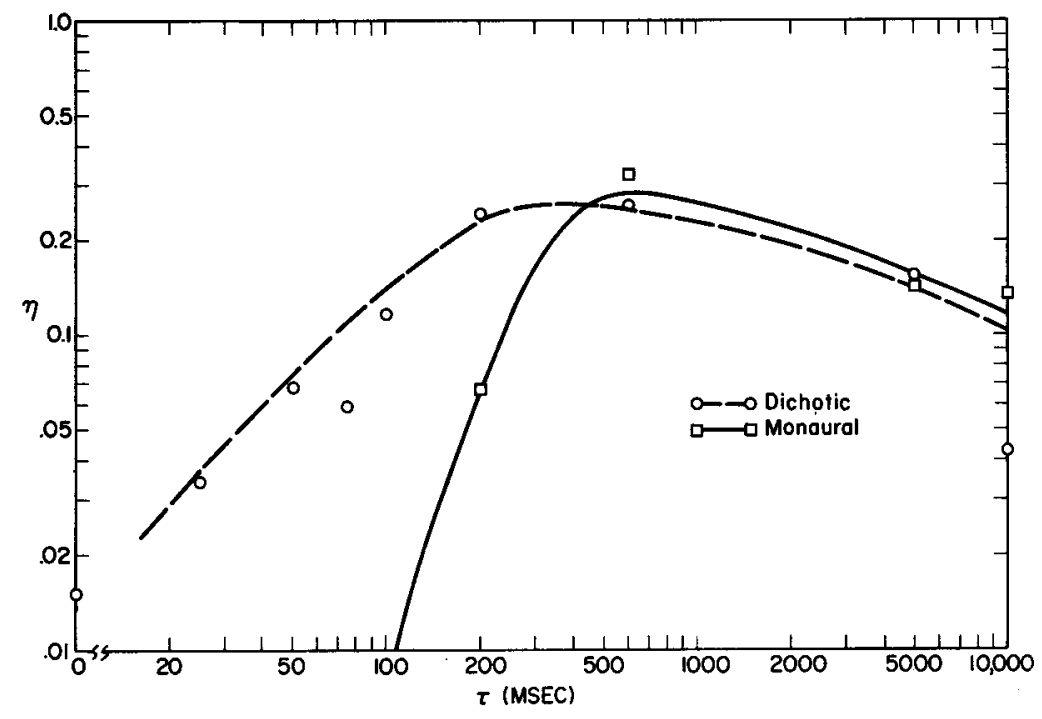

Fig. 3, $\eta$ as a function of $\tau$ for observer 2 . 


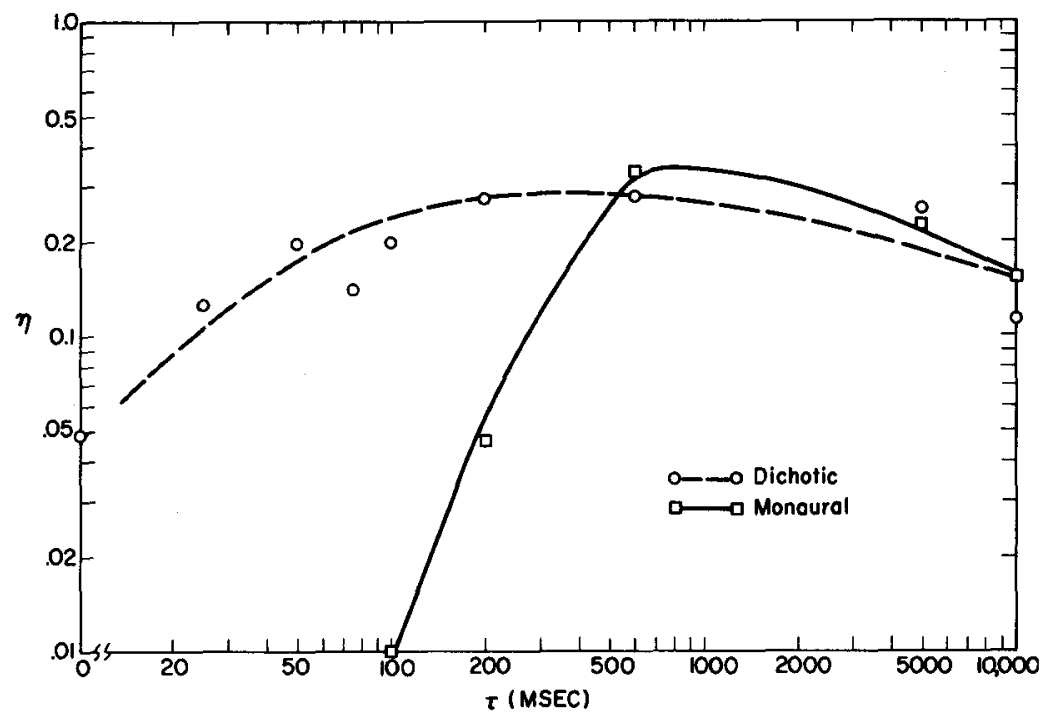

Fig. 4. $\eta$ as a function of $\tau$ for observer 3 .

of the interference functions. If the binaural presentation succeeds in partially isolating the inputs until after the peripheral filtering, one will observe only a moderate drop-off in performance (compared to that of the monaural task) as the temporal separation of the intervals is reduced to zero. Some drop-off in performance, however, would be expected as a consequence of the more central operation of a selective processing system. Consideration of the monaural functions, together with the dichotic, might allow greater specification of the filter-like character of the system at the end organ.

Method

The first task was a two-alternative forced-choice task, presented monaurally, which employed two 50 msec., random amplitude, $1000 \mathrm{cps}$ signals, $\mathrm{S}$ and S+ $\Delta V$, where $\Delta V$ was a fixed amplitude. Two observation intervals were marked visually, and the signals were presented in the intervals in random order. The observer was to report whether he thought the $(S, S+\Delta V)$ or
(S+ $\Delta V, S)$ configuration had been presented, that is, which interval contained the larger amplitude signal. White Gaussian noise was present continuously in the PDR-8 earphone. In the second task, a comparison dichotic task, the observation intervals were further isolated by channelling each input interval to a specified ear. The observer was told that the first signal-interval would always be presented to the leftear and the second signal-interval to the right; he was again asked to report which interval, 1.e. which ear, contained the larger amplitude signal. When the two signal-intervals occur simultaneously in time, the observer's task thus becomes one of discriminating which ear, rather than which interval, contained the larger amplitude signal. Again, uncorrelated white noise was added to each input. The monaural and the dichotic tasks were run at various $\Delta V^{\prime}$ 's and a wide range of time intervals, $r$, between the ending time of the first signal and the ending time of the second. The performance measure employed was the observer efficiency; $\eta$, which is

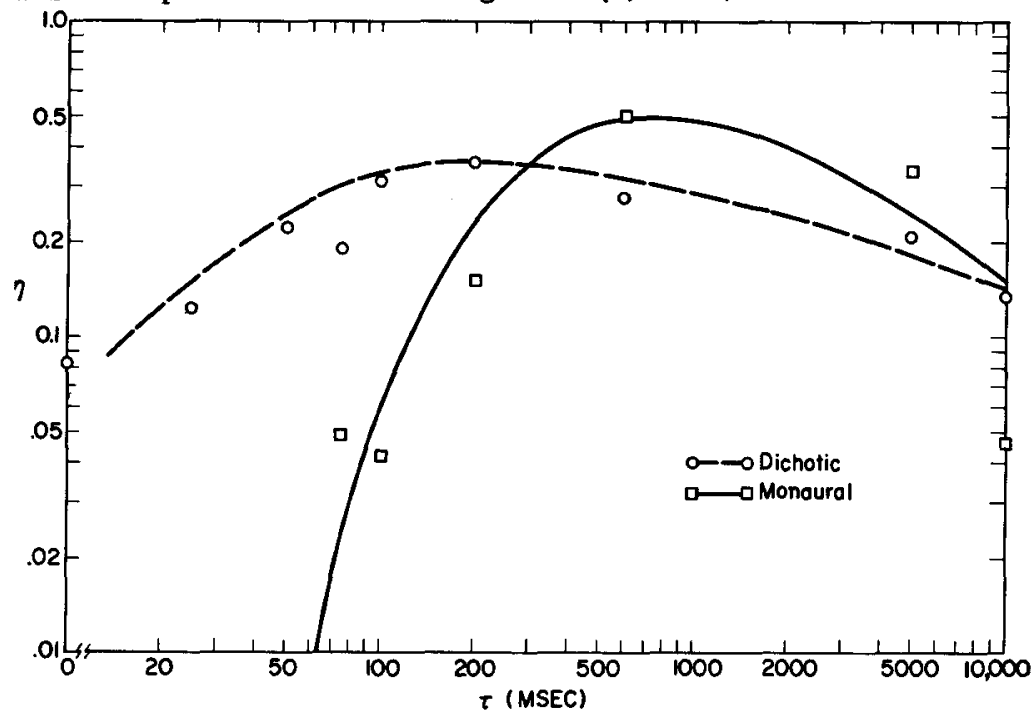

Fig. 5. $\eta$ as a function of, for observer 4. 
defined as the ratio of the energy required by a theoretical ideal observer to that required by an unknown observer, under conditions of equivalent performance. Tanner (1958) describes the theory of the ideal observer and the requisite assumptions for the use of the efficiency measure. For all conditions, $\eta$ was found to be independent of signal level. The earphone voltages employed for $S$ varied between $.008 \mathrm{~V}$ and $.014 \mathrm{v}, \Delta V^{\prime}$ 's employed were between .004 and $.008 \mathrm{v}$. For a $\Delta \mathrm{V}$ of $.006 \mathrm{v}, 2 \mathrm{E} / \mathrm{N}_{\mathrm{O}}$ was equal to 10.3. All tonal signals were gated on and off at positive zero crossings; approximately 1200 trials per condition were run.

\section{Resulis and Discussion}

Plots for all four observers of $\log \eta$ versus $\log r$ are shown in Figs. 2 through 5 . At $r$ 's greater than 1 sec., the monaural and the dichotic functions appear to begin converging. At $r$ less than $1 \mathrm{sec}$. there is great disparity in the functions. The monaural functions appear to peak slightly later than the dichotic, and can be seen to drop off towards chance as the separation between the signals goes to zero $(r=50$ msec.). The dichotic functions drop off less steeply to a finite $\eta$ when the signals completely overlap, an indication in some measure of the efficiency of lateralization performance.

That the dichotic functions do drop smoothly to a finite $\eta$, is support for the idea that one will find filter effects higher up in the system than at the end organ itself. Attempts to fit the dichotic function (at $r$ less than 100 msec.) with a single time constant filter model were not successful, but the time constants which result from such a procedure are of a magnitude consistent with those reported in other studies. Attempts to extract the end organ time constant, for the purpose of comparison with electrophysiological studies or ear-impedance data, are probably also premature. Yet the demonstration that one may separate out some of the effects of more "central" filter processes is felt to be of value, and the procedure may be useful in quite different sorts of tasks. In experiments involving cue signals, for example, it may be possible to minimize end organ interference by using contralateral rather than ipsilateral cue inputs. Combining this approach with recognition of the memory effects noted earlier, one might arrive at something like an optimal time separation between cue inputs and signal inputs, thereby allowing maximum opportunity for any cue effects to be observed. 3

\section{References}

Tanner, Wilson P, Jr. Definitions of $d^{\prime}$ and $\eta$. J. Acoust. Soc. Amer., 1958, 30, 922-928.

Tanner, Wilson P., Jr. Physiological implications of psychophysical data. Ann. N. Y. Acad. Sci., 1961, 89, 752-765.

\section{Notes}

1. This research was supported by the Advanced Research Projects Agency, Assistant Director, Behavioral Sciences, Command and Control Research, and was monitored by Behavioral Sciences Division, Air Force Office of Scientific Research, under Contract AF $49(638)-1233$.

2. Now at Purdue University, Lafayette, Indiana.

3. The reader is directed to the following general references. Detection Theory: Swets, J. A. (Ed.), Signal detection and recognition by human observers, New York: Wiley, 1964. Related Binaural Signal Detection: Vector Theory, Chapter 15 in Foundations of modern auditory theory. J. V. Tobias and E. D. Schubert (Eds.), New York: Academic Press (to be published). 